\title{
LOS ALUMNOS PREFIEREN DIFERENTES ESTRATEGIAS DIDÁCTICAS DE LA ENSENANZA DE LAS CIENCIAS EN FUNCIÓN DE SUS CARACTERÍSTICAS MOTIVACIONALES
}

\author{
MARTÍN DÍAZ, M.J. ${ }^{(1)}$ y KEMPA, R.F.(2) \\ (1) I.B. Antonio Machado, Madrid. \\ (2) Departament of Education. University of Keele. Keele. Staffordshire ST5 5BG, Inglaterra.
}

\section{SUMMARY}

We carried out empirical research into the relationship between students' motivational patterns and their preferences for different teaching procedures in science education. According to the results obtained, students tend to prefer those teaching procedures and strategies that allow their motivational needs to be met and reject those conflicting with them.

\section{INTRODUCCIÓN}

La selección de estrategias didácticas representa un importante paso del diseño curricular. Por estrategias didácticas se entienden Ios métodos utilizados en el aula para lograr que los alumnos alcancen unos ciertos conocimientos y habilidades. Este término encierra una amplia gama de actividades, a través de las cuales se desarrolla la interacción profesor-alumnos en las clases.

La mayoría de los proyectos curriculares incorporan recomendaciones sobre cómo se debe conducir la enseñanza, influyendo o prescribiendo las estrategias didácticas que los profesores deben utilizar. Un estudio comparativo sobre 45 proyectos curriculares lleva a Heathcote, Kempa y Roberts (1982) a concluir que «está justificado decir que las decisiones tomadas sobre las estrategias didácticas tal y como aparecen en los proyectos curriculares fueron casi enteramente hechas sobre bases pragmáticas y creeencias firmes».

Tomando como ejemplo la dimensión que presenta en sus extremos el aprendizaje por descubrimiento y' Ia enseñanza por tansmisión verbal, es importante señalar que, en los estudios empíricos realizados por Kaufman (1971) y Herman (1969) sobre los méritos relativos de ambas estrategias, estos autores concluyen que los resul- tados son conflictivos y poco concluyentes, pero que la polémica puede continuar durante años, como ya hemos podido comprobar.

Uno de los puntos importantes cuando se entra en discusión sobre las ventajas y desvantajas de diferentes estrategias de enseñanza es su capacidad motivadora sobre los alumnos. Bruner (1961), un fuerte abogado defensor del aprendizaje por descubrimiento, señala que este procedimiento es más motivante que la enseñanza por transmisión verbal, mientras que Ausubel (1963) adopta la posición contraria.

Nosotros consideramos que el punto clave de este y otros debates en la misma línea es que generalmente los diferentes autores hablan de los méritos motivantes de un determinado método de enseñanza, aplicándolo a todos los alumnos en cualquier situación. Como Hofstein y Kempa (1985) han señalado, «los autores generalmente parecen asumir que las cualidades motivantes de una estrategia en particular son una función intrínseca de tal estrategia".

La posición defendida en este artículo es que las características individuales de los alumnos se deben tener en 
cuenta si realmente deseamos mejorar el proceso ensenanza-aprendizaje. Los mejores materiales didácticos son inútiles si Ios alumnos no están interesados en ellos, y las mejores estrategias didácticas son inefectivas si los alumnos no están motivadas por ellas.

Se han desarrollado algunos modelos teóricos (Hofstein y Kempa 1985, Good y Power 1976, Dodge 1978) en Ios que se Ilevan a cabo un "emparejamiento» (matching) entre las características motivacionales de los alumnos y algunas variables instruccionales. La posición defendida por Hofstein y Kempa (1985) es que los alumnos poseen determinadas preferencias por diferentes formas de aprendizaje o métodos de enseñanza, y que dichas preferencias están basadas en su propio «modelo motivacional».

El concepto de «modelo motivacional» es introducido por Adar (1969), quien, estudiando los diferentes motivos onecesidades que conducen a los alumnos en su aprendizaje, identifica cuatro tipos de necesidades en ellos: necesidad de obtener éxito, necesidad de satisfacer la propia curiosidad, necesidad de cumplir las obligaciones y necesidad de relacionarse con los demás. Según el predominio de uno de estos «motivos», los alumnos se pueden clasificar en: alumnos que buscan el éxito, alumnos curiosos, alumnos concienzudos y alumnos sociables.

Tomando esto como base, Hofstein y Kempa (1985) desarrollan un modelo teórico en el que asignan diferentes estrategias de enseñanza de las Ciencias a los cuatro modelos motivacionales descritos por Adar. El trabajo presentado en este artículo representa un intento para dar validez empírica a este modelo.

\section{MATERIALES Y MÉTODOS}

\section{Instrumentos de medida}

Se diseñaron y administraron tres tipos de cuestionarios, todos ellos bajo el formato Likert, destinados a conocer: - los modelos motivacionales de los alumnos - las preferencias de los alumnos por diferentes métodos de enseñanza de las Ciencias

- las experiencias de los alumnos en dichos métodos.

\section{a) Cuestionario sobre los modelos motivacionales (CMM)}

Este cuestionario constaba de 60 íterns, que intentaban recoger las características de los cuatro modelos motivacionales descritos, los cuales constituyeron las dimensiones del cuestionario. Cada ítem estaba constituido por una frase que reflejaba una característica motivacional. Los alumnos tenían que responder, en una escala de 5 puntos, si era verdadero o falso lo que dichas frases decían, cuando se las aplicaban a si mismos. Los ítems de este cuestionario se presentan en el Anexo.

b) Cuestionario sobre las preferencias de los alumnos por diferentes métodos de enseñanza de las Ciencias (CPMEC)
Este cuestionario constaba de 80 ítems, que recogían las siguientes dimensiones y subdimensiones:

1. Forma de adquisición del conocimiento:

la. enseñanza formal por transmisión verbal

Ib. aprendizaje por descubrimiento

Ic. uso de libros de referencia para la obtención de información.

2. Trabajo práctico:

2a. trabajo práctico versus explicaciones teóricas.

2b. trabajo práctico con instrucciones claras y precisas dadas por el profesor.

3. Control del profesor versus control del alumno:

3a. oportunidad dada a los alumnos para seguir su propia iniciativa.

4. Organización de las actividades del aprendizaje:

4a. trabajo individualizado

4b. trabajo en pequeños grupos.

5. Evaluación:

5a, evaluación constante por parte del profesor

5b. rechazo a ser evaluado.

Esta clasificación de los métodos de enseñanza de las Ciencias está basada en la realizada por Eggleston, Galton y Jones (1976) y Heathcote, Kempa y Roberts (1982), con la adición de algunas dimensiones como son «evaluación» y "organización de las actividades del aprendizaje». Este cuestionario no se presenta debido a que no queremos alargar innecesariamente este artículo.

Un peligro potencial que lo acompañaba era que los alumnos podían ser preguntados sobre estrategias de enseñanza que podían ser poco familiares o incluso desconocidas para ellos, y por tanto sus preferencias podían estar distorsionadas o disfrazadas. Para superar esta difícultad, administramos a los alumnos un tercer tipo de cuestionario destinado a conocer sus experiencias en los métodos de enseñanza antes mencionados.

Los resultados de las correlaciones entre experiencias y preferencias de los alumnos por dichos métodos han sido reportados previamente (Martín Díaz 1989).

\section{Muestra}

Participaron en la experiencia 390 alumnos/as de $2^{\circ}$ de BUP, pertenecientes a cinco institutos de Bachillerato, dos de la provincia de Madrid y tres de la de Badajoz.

\section{Análisis estadísticos}

Los análisis estadísticos se realizaron con la ayuda đel paquete estadístico SPSSX, que es una versión última y más potente del SPSS (Statisticai Package for Social 
Scientists). Dichos análisis comprendieron estudios de fiabilidad (cálculo de los coeficientes de Cronbach) y validez (análisis factorial), de correlación, análisis de varianza y covarianza, etc.

\section{RESULTADOS}

\section{Estudios de fiabilidad y validez de los cuestio- narios}

Los resultados de estos análisis han sido previamente descritos (Martín Díaz 1989, Kempa y Martín Díaz 1990). Sólo es interesante señalar que en el cuestionario sobre modelos motivacionales (CMM) se mantuvieron las dimensiones establecidas, y se fijaron claramente los perfiles de dichos modelos, que aparecieron en total consonancia con Ios expuestos por Adar (1969) y Orbach (1979), y que pasamos a describir brevemente.

Los alumnos curiosos exhiben las siguientes características:

- Gtan interés en aprender sobre nuevos sucesos o fenómenos científicos, incluso sobre aquéllos que no aparecen en los libros de texto.

- Inclinación a examinar, explorar y manipular la información.

- Obtención de satisfacción como consecuencia de esta exploración y manipulación.

- Búsqueda de complejidad en las actividades escolares.

Los alumnos concienzudos se caracterizan por:

- Deseo de hacer aquello que está bien y evitar lo que está mal.

- Incapacidad para saber cuando han cumplido perfectamente con sus obligaciones.

- Necesidad de soporte externo.

- Desarrollo de sentimientos de culpabilidad frente a cualquier incapacidad.

- Falta de confianza en sí mismos o intolerancia ante los errores cometidos.

Los alumnos sociables presentan las siguientes características:

- Necesidad de conseguir y mantener buenas relaciones de amistad con los compañeros.

- Muy buena disposición para ayudar a los compañeros en todas las actividades escolares.

- Ningún temor a "fallar» en las situaciones escolares orientadas hacia el éxito acádemico.

- Concesión de mayor importancia a las relaciones de amistad que a las actividades y factores escolares.
Los alumnos que buscan el éxito se caracterizan:

- Preferencia por situaciones competitivas.

- Necesidad de obtener éxito en dichas situaciones.

- Necesidad de conseguir estima y prestigio del profesor y del resto de los compañeros, como consecuencia de la victoria en aquéllas.

En cuanto al cuestionario CPMEC el análisis factorial no aportó ninguna modificación en las dimensiones anterormente descritas digna de mención.

En todos los análisis posteriores se utilizaron las dimensiones tal y como quedaron definidas por Ios estudios de fiabilidad y validez.

\section{Estudio de los modelos motivacionales. Correla- ciones y distribución}

Es posible que se piense que los modelos motivacionales no tienen por qué ser «puros», es decir, en nuestros alumnos pueden «cohabitar» varias de las necesidades que les dirigen hacia el aprendizaje. Adar (1969) indica que existen diferentes tipos de alumnos según el «predominio» de una de estas necesidades.

Nuestros resultados corroboran esta idea como puede observarse en la tabla I. Son especialmente remarcables las siguientes correlaciones: los alumnos curiosos y los concienzudos están correlacionados positivamente, los alumnos sociables presentan una doble correlación negativa con los alumnos que buscan el éxito y con los concienzudos, y, finalmente, los alumnos que buscan el éxito presentan una cierta correlación positiva con los concienzudos.

Aunque las correlaciones anteriores tienen valores bajos, todas ellas son estadísticamente significativas a un nivel del $0.1 \%$, y podemos asegurar que no son fuente de sorpresas.

En este punto nos podemos preguntar jcuál es la distribución de ruestros alumnos en los diferentes modelos motivacionales? La respuesta a esta pregunta la encontramos en la Figura 1, donde se presenta la distribución

Tabla I

Correlaciones entre los modelos motivacionales

\begin{tabular}{lcccc}
\hline MODEIIO & \multicolumn{4}{c}{ MODELOS MOTNACIONALES } \\
& BUSC. EXTTO & CURIOSO & CONCIENZUDO & SOCLABLE \\
\hline BUSC.EXITO & 1.000 & .071 & $.202^{*}$ & $-.239^{*}$ \\
CURIOSO & & 1.000 & $.370^{*}$ & .014 \\
CONCIENZUDO & & & 1.000 & $-.175^{*}$ \\
SOCIABLE & & & & 1.000
\end{tabular}

* Nivel de significasion estadística: "p<0.001 
de los alumnos en los «niveles máximos» đe cada uno de los tipos de motivación (para mayor información sobre la metodología seguida ver Martín Diaz 1989). Las cuatro primeras columnas representan el \% de alumnos respecto a toda la población estudiada que tienen un «nivel máximo» de motivación por curiosidad, concienciación, afiliación y búsqueda de éxito respectivamente. Las columnas 4-8 representan el \% de alumnos que tiene niveles máximos en dos tipos de motivación simultáneamente, y así sucesivamente.

Es importante destacar que casi la mitad $(43 \%)$ de la población estudiantil analizada tiene características motivacionales tales que pertenece a los grupos con «niveles máximos» de Ios cuatro típos de motivacióni considerados. Asimismo, consideramos interesante señalar que el \% de alumnos que presentan simultáneamente "niveíes mínimos» en los cuatro tipos de motivación es un $0 \%$, es decir, todos los alumnos alcanzan un cierto nivel en alguno de ellos.

\section{Análisis de covarianza}

El análisis de covarianza nos permitió estuđiar las variaciones en las preferencias de los alumnos por diferentes estrategias de la enseñanza de las Ciencias en función de los niveles de los cuatro tipos de motivación. La existencia de correlaciones entre los modelos motivacionales nos exigió la utilización de los análisis đe covarianza, en

figura 1

Distrihución de la pohtación en lox míximes niveles de moxivación.

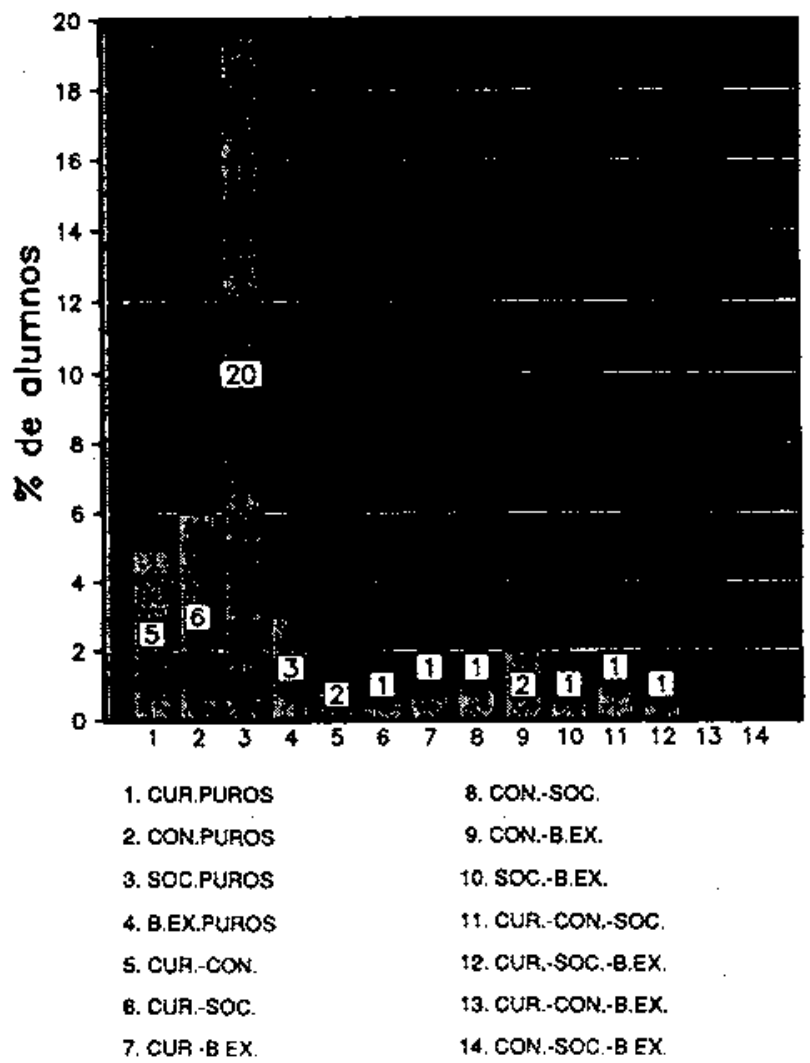

lugar de los de varianza (Martín Díaz 1989). Se eligieron sucesivamente cada modelo motivacional como variable independiente y los tres modelos restantes como «covariantes», siendo las preferencias por los métodos de enseñanza las variables dependientes. Para la partición de los alumnos en grupos con diferentes niveles de motivación se siguió un criterio basado en la puntuación obtenida en las diferentes dimensiones del cuestionario CMM (Martín Díaz 1989). Por lo tanto, presentamos los resultados para cada uno de los modelos motivacionales, sucesivamente:

\section{Alumno curioso}

Los alumnos curiosos muestran una clara preferencia por las siguientes estrategias didácticas (ordenadas en orden decreciente de los valores de F) (Tabla H): aprendizaje por descubrimiento, uso de libros de referencia para la obtención de información, trabajo práctico versus explicaciones teóricas, oportunidad para seguir su propia iniciativa. $Y$ muestran un rechazo por: trabajo práctico con instrucciones claras y precisas, y enseñanza formal por transmisión verbal.

Estos datos tomados globalmente nos indican que los alumnos curiosos prefieren estar «envueltos activamente» en su aprendizaje. Les gusta descubrir, buscar información, tomar decisiones, etc. No quieren ser meros «receptores de información», no necesitan unos objetivos claramente especificados o instrucciones precisas, por el contrario prefieren un aprendizaje que requiere su participación activa, que les exige manipular información, juzgar y decidir.

La preferencia mostrada por el aprendizaje por descubrimiento y uso de libros de referencia está en total concordançia con las características de estos alumnos, ya que

Tabla II

\begin{tabular}{|c|c|c|c|c|c|}
\hline \multicolumn{6}{|c|}{ 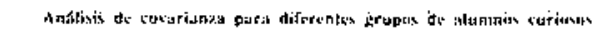 } \\
\hline \multirow{3}{*}{$\begin{array}{l}\text { ISTRATIGLA } \\
\text { I,IISACTECA }\end{array}$} & \multicolumn{4}{|c|}{ 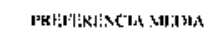 } & \multirow{3}{*}{ | 1 | } \\
\hline & & & \&:Ix]s & & \\
\hline & $\begin{array}{l}\text { Dijkr } \\
\text { Mustar } \\
\text { 26s" }\end{array}$ & 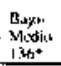 & 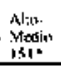 & 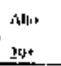 & \\
\hline 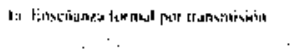 & 264 & 2.61 & 241 & 2.3 .3 & +1.76 \\
\hline 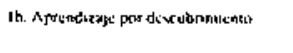 & 30 & $i, 8,4$ & 412 & 0.42 & $\begin{array}{l}19.6 .8 \\
1(x, 1)]\end{array}$ \\
\hline Ic. lino es libson de referencia & 2.08 & 2.12 & 2.43 & $2 . \mathrm{N}$ & $\begin{array}{l}\text { 12.us } \\
\text { 1000ib] }\end{array}$ \\
\hline 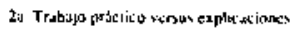 & .3 .45 & 3 si & 3.34 & 1.04 & $\begin{array}{l}6.43 \mid \\
{[.001+1]}\end{array}$ \\
\hline 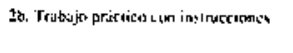 & 4.23 & 4.0 .3 & $.6 \mathrm{~K}$ & 132 & $\frac{10.68}{1.6 \times 101}$ \\
\hline 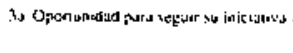 & .3 .55 & $3 \times 7$ & 405 & 4.14 & (ans \\
\hline 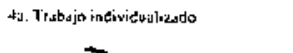 & 2.76 & 2.32 & 278 & 2.k? & $\begin{array}{l}112 \\
{\left[.4(1), x_{1}\right.}\end{array}$ \\
\hline 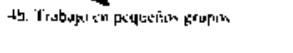 & $3 \mathrm{Bt}$ & 3.96 & $3.8,3$ & 4.! & 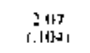 \\
\hline 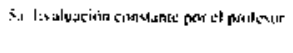 & s.us & $3 . \mathrm{B3}$ & 340 & 3 & 1.34 \\
\hline 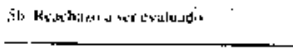 & 33 & 1.13 & 390 & $\therefore 4$ & $\begin{array}{c}74 \\
1304 \\
\end{array}$ \\
\hline
\end{tabular}

ENSEÑANZA DE LAS CIENCIAS, 1991, 9 (1) 
ambos métodos proporcionan al alumno curioso la posibilidad de manipular información y le sirven como estímulo de su propia curiosidad, punto básico para lograr su motivación.

Estas preferencias así como la preferencia por situaciones que les den la oportunidad de seguir su propia iniciativa han sido previamente postuladas (Hofstein y Kempa 1985, Orbach 1979). En relación a la última de ellas, Orbach indica que las actividades para este tipo de alumnos «deben ser elegidas, definidas y diseñadas por los alumnos mismos antes que por el profesor».

En relación con el trabajo práctico, los alumnos muestran una preferencia por él, pero siempre y cuando no vaya acompanado de unas instrucciones claras y precisas, siempre y cuando sea "abierto» en términos del procedimiento a seguir, es decir, que permita a los alumnos expresar y satisfacer su propia curiosidad.

De la misma forma, no es sorprendente que estos alumnos muestren un rechazo hacia aquellos métodos de enseñanza totalmente centrados en el profesor, donde éste es la figura activa y a ellos les queda reservado un papel pasivo.

\section{Alumno concienzudo}

Los resultados del análisis de covarianza con la escala «alumno concienzudo» como variable independiente se muestran en la tabla III. Existen variaciones estadísticamente significativas con el nivel de motivación por concienciación en la preferencia por las siguientes estra-

Tábla III

\begin{tabular}{|c|c|c|c|c|c|}
\hline \multicolumn{6}{|c|}{ Anśtisis de covarianza para diferentes grupos de alumnos concienzudos } \\
\hline \multirow{2}{*}{$\begin{array}{l}\text { ESTRATEGIA } \\
\text { DIDACITA }\end{array}$} & \multicolumn{4}{|c|}{ PREFERENCIA MEDIA } & \multirow{2}{*}{$\begin{array}{c}\text { F } \\
\text { (Signif.) }\end{array}$} \\
\hline & $\begin{array}{l}\text { Bajo } \\
\text { Mtedio } \\
21=\end{array}$ & $\begin{array}{l}\text { GR } \\
\text { Bajo- } \\
\text { Medio } \\
\text { 109." }\end{array}$ & $\begin{array}{l}\text { RUPOS } \\
\text { Alto } \\
\text { Medio } \\
175^{*}\end{array}$ & $\begin{array}{l}\text { Alto } \\
37 *\end{array}$ & \\
\hline 1a. Enseñanza formal por transmision & 2.23 & 2.49 & 2.52 & 2.63 & $\begin{array}{c}2.29 \\
(.078)\end{array}$ \\
\hline 1b. Aprendizaje por descubrimiento & 4.07 & 3.96 & 3.98 & 4.09 & $\begin{array}{c}.97 \\
(.404)\end{array}$ \\
\hline 1c. Uso de tibros de referencia & 2.72 & 2.34 & 2.28 & 2.09 & $\begin{array}{c}3.88 \\
(.010)\end{array}$ \\
\hline 2a. Trabajo práctico versus explicaciones & 3.93 & 3.64 & 3.62 & 3.65 & $\begin{array}{c}1.24 \\
(.295)\end{array}$ \\
\hline 2b. Trabajo priftico con instrucciones & 3.12 & 3.81 & 3.86 & 4.14 & $\begin{array}{c}7.68 \\
(.000)\end{array}$ \\
\hline 3a. Oportuntidad para seguir su iniciativa & 4.27 & 3.92 & 3.92 & 4.00 & $\begin{array}{c}1.64 \\
(.179)\end{array}$ \\
\hline 4a. Trabajo individualizado & 3.04 & 2.65 & 2.77 & 2.87 & $\begin{array}{c}1.93 \\
(.125)\end{array}$ \\
\hline 4b. Trabajo en pequerios grupos & 3.72 & 3.79 & $3.94^{\circ}$ & 4.21 & $\begin{array}{c}3,46 \\
(0.17)\end{array}$ \\
\hline 5a. Evaluacior constante por el profesor & 3.20 & 3.70 & 3.98 & 4.26 & $\begin{array}{l}12.87 \\
(.000)\end{array}$ \\
\hline 5b. Reachazo a ser evaluado & 3.46 & 3.33 & 3.35 & 3.35 & $\begin{array}{c}.15 \\
(.929)\end{array}$ \\
\hline
\end{tabular}

* Número de alurnos. Las preferencias mesias están basadas en una escala de cinco puntos, que varia desde $5=a l t a$ preferencia hăsta 1 =baja preferencia. tegias didácticas (en orden decreciente de los valores de F): evaluación por parte del profesor, trabajo experimental con instrucciones claras y precisas, uso de libros de referencia para la obtención de información y trabajo en pequeños grupos.

Además, existe una relación positiva con la enseñanza formal por transmisión verbal, con un valor de p próximo al criterio normalmente aceptado de $\mathrm{p}<0.05$. Se debe notar que sólo en el caso del uso de libros de referencia, los alumnos concienzudos muestran rechazo.

Estos resultados indican que los alumnos concienzudos prefieren métodos de enseñanza con unos claros y precisos objetivos e instrucciones. Desean fuertemente ser evaluados con frecuencia por sus profesores. Es decir, el profesor juega un papel muy importante para ellos, ya que debe darles el soporte externo que necesitan a causa de su incapacidad para saber lo que está bien y lo que está mal, y también debe darles las instrucciones que necesitan para hacer lo que está bien y evitar lo que está mal. ¡Aprender, para estos estudiantes, es una obligación!

Estos alumnos no muestran ni rechazo ni preferencia por el aprendizaje por descubrimiento. Se podría concluir que esta estrategia puede ser utilizada con los alumnos concienzudos, siempre que esté altamente guiada, es decir, que sea un descubrimiento muy dirigido. Sin embargo, sí que deben ser evitadas aquellas actividades que sean «abiertas» en términos de los procedimientos a seguir para realizarlas.

Estos datos están en total armonía con las estrategias postuladas como ideales para los alumnos concienzudos (Hofstein y Kempa 1985, Orbach 1979, Good y Power 1976). Por ejemplo, Orbach (1979) escribe: «Es bastante posible que el viejo método de enseñanza frontal sea motivacionalmente mejor que alguno de los más modernos métodos de instrucción». Sin embargo, lo que no es postulado por estos autores es la preferencia creciente por el trabajo en grupo con el nivel de motivación por concienciación. Es posible que los alumnos concienzudos vean en sus compañeros de grupo una fuente de soporte en el cumplimiento de sus obligaciones y tareas del aprendizaje.

\section{Alumno sociable}

Los resultados del análisis de covarianza, cuando la escala «alumno sociable» es utilizada como variable independiente y el resto de los modelos motivacionales como covariantes, se presenta en la tabla IV. Es impor* tante indicar que el criterio utilizado para la partición de los alumnos en función de su nivel de motivación por afiliación es diferente al utilizado en el resto de los casos (Martín Díaz 1989).

Podemos observar variaciones estadísticamente significativas con el nivel de motivación por afiliación en las preferencias por las siguientes estrategias de enseñanza de las Ciencias (en orden decreciente de los valores de F): oportunidad de seguir su propia iniciativa, trabajo individualizado, rechazo a ser evaluado, trabajo en pe- 


\section{Tarkia IV}

Análisis de covarianza para díferentes grupos de alumnos sociables

\begin{tabular}{|c|c|c|c|c|c|}
\hline \multirow{2}{*}{$\begin{array}{l}\text { ESTRATEGIA } \\
\text { DIDACTICA }\end{array}$} & \multicolumn{4}{|c|}{ PREFERENCIA MEDIA } & \multirow{2}{*}{$\begin{array}{c}\text { F } \\
\text { (Signif, })\end{array}$} \\
\hline & $\begin{array}{l}\text { Bajo } \\
\text { Modio } \\
80^{*}\end{array}$ & $\begin{array}{l}\quad \text { GR } \\
\text { Bajo- } \\
\text { Modio } \\
83^{*}\end{array}$ & $\begin{array}{l}\text { UPOS } \\
\text { Alio } \\
\text { Medio } \\
101^{*}\end{array}$ & $\begin{array}{l}\text { Alto } \\
78 * \\
\end{array}$ & \\
\hline 1a. Enserianza formal por transraision & 2.72 & 2.54 & 2.38 & 2.36 & $\begin{array}{c}7.79 \\
(.000)\end{array}$ \\
\hline Ib. Aprendizaje por descubrimiento & 3.82 & 3.99 & 4.08 & 4.05 & $\begin{array}{c}5.32 \\
(.001)\end{array}$ \\
\hline 1c. Uso de libros de referencia & 2.31 & 2.17 & 2.45 & 2.27 & $\begin{array}{c}3.02 \\
(.030)\end{array}$ \\
\hline 2a. Trabajo práctico versus explicaciores & 3.44 & 3.68 & 3.66 & 3.82 & $\begin{array}{c}4.20 \\
(.006)\end{array}$ \\
\hline 2b. Trabajo práctico con instrucciones & 3.95 & 3.82 & 3.84 & 3.70 & $\begin{array}{c}1.36 \\
(.256)\end{array}$ \\
\hline 3a. Oportunidad para seguir su inticiativa & 3.54 & 3.79 & 4.17 & 4.28 & $\begin{array}{l}16.48 \\
(.000)\end{array}$ \\
\hline 4a. Trabajo individualizado & 3.16 & 2.81 & 2.64 & 2.45 & $\begin{array}{l}11.60 \\
(.000)\end{array}$ \\
\hline ab. Trabajo en pequeños grupos & 3.63 & 3.78 & 4.05 & 4.16 & $\begin{array}{c}9.80 \\
(.000)\end{array}$ \\
\hline Sa. Evajuación constante por $t$ l profesor & 3.94 & 3.98 & 3.77 & 3.81 & $\begin{array}{c}1.96 \\
(.119)\end{array}$ \\
\hline Sb. Reachazo a ser evaluado & 3.02 & 3.19 & 3.46 & 3.69 & $\begin{array}{l}10.16 \\
(000)\end{array}$ \\
\hline
\end{tabular}

* Número de ailumnos. Las preferencias medias estan basadas en una escala de cinco puntos, que vasia desde $S=a$ alta preferencia hasta $1=$ baja preferencia

quetios ytupos, enschanci lormal por trusimision verbal, aprendizaje por descubrimiento y trabajo práctico versus explicaciones teóricas. Todas estas variaciones significan niveles crecientes de preferencia, a excepción de dos variables: trabajo individualizado y enseñanza formal, que son rechazados por los alumnos sociables. Tambien se pueden observar algunos efectos marginales en «uso de libros de referencia», que no merecen una especial atención.

No es sorpredente, la preferencia exhibida por estos alumnos por el trabajo en grupo. Son numerosos los autores (Hofstein y Kempa 1985, Orbach 1979, Dodge 1978, Good y Power 1976) que han argumentado que este trabajo en grupo es una de las mejores vías para lograr su motivación. No solamente prefieren trabajar en grupo, sino que exhiben un fuerte rechazo por el trabajo individualizado.

Las preferencias moderadas de estos alumnos por el aprendizaje por descubrimiento y por el trabajo práctico se pueden explicar en base a la oportunidad que dichos métodos les dan para la interacción social. Su experiencia sobre estas estrategias les dice que ambas suelen llevarse a cabo en pequeños grupos.

No obstante, otra explicación es posible. Como hemos indicado y ha sido señalado por Addar (1969) y Orbach (1979), los alumnos sociables están libres del miedo a fallar en situaciones orientadas al éxito académico, sus propias características les llevan a inclinarse hacia climas de aprendizaje no autoritarios, en los cuales pueden organizar su propio aprendizaje. Esto explica que los alumnos quieran tener la oportunidad de seguir su propia iniciativa y rechacen ser evaluados. Asimismo, no cabe duda de que en las estrategias anteriormente nombradas, los alumnos gozan de un mayor grado de libertad que en aquellas otras más formales y totalmente centradas en la figura del profesor, y por tanto, son preferidas por estos alumnos.

\section{Alumno que busca el éxito}

Como puede observarse en la tabla V, sólo presenta variación con el nivel de motivación por búsqueda del éxito el nivel de preferencia por los siguientes métodos de enseñanza de las Ciencias: aprendizaje por descubrimiento, oportunidad de seguir su propia iniciativa y enseñanza formal por transmisión verbal.

Ha sido ampliamente difundido en la literatura que los alumnos motivados por la obtención de éxito tienden a identificarse habitualmente con actividades que encierran un grado medio de dificultad, y raramente, con un alto grado de la misma (McClelland et al. 1953, McCleliand 1989, Orbach 1979, Clifford, 1988). Otros autores, yendo más lejos, indican que este tipo de alumnos eligen actividades fáciles en su comienzo, para seleccionar actividades de dificultad creciente, una vez que han tenido éxito en las primeras (Schneider y Pose 1982 , Kuhl y Blankenship 1979). Detrás de estas ideas subyace que la probabilidad del éxito es siempre una función de la dificultad de la actividad. Asimismo, cuanta más dificulıad encierra ésta, mayor es Ia probabilidad del fracaso. Y también, cuanta mayor es la dificultad percibida por el alumno, mayor satisfación acompañatá al

Tabla V

Análisis de covarianza para diferentes grupos de aiumnos buscadores de exite

\begin{tabular}{|c|c|c|c|c|c|}
\hline \multirow{2}{*}{$\begin{array}{l}\text { ESTRATEGIA } \\
\text { DDACTICA }\end{array}$} & \multicolumn{4}{|c|}{ PREFERENCIA MEDLA } & \multirow{2}{*}{$\begin{array}{c}\text { F } \\
\text { (Signif.) }\end{array}$} \\
\hline & $\begin{array}{l}\text { Bajo } \\
\text { Medio } \\
61^{*} \\
\end{array}$ & $\begin{array}{l}\text { GRL } \\
\text { Bajo- } \\
\text { Madio } \\
143^{*}\end{array}$ & $\begin{array}{l}\text { UPOS } \\
\text { Alto } \\
\text { Modio } \\
119^{*} \\
\end{array}$ & $\begin{array}{l}\text { Alto } \\
21^{*}\end{array}$ & \\
\hline 1a. Enseñanza formal por transmisión & 2.58 & 2.53 & 2.49 & 2.17 & $\begin{array}{c}3.49 \\
(016)\end{array}$ \\
\hline ib. Agrendizaje por descubrimiento & 3.80 & 3.99 & 4.05 & 4.14 & $\begin{array}{c}4.27 \\
(.006)\end{array}$ \\
\hline le. Uso de libros de referencia & 2.24 & 2.29 & 2.36 & 2.34 & .45 \\
\hline 2a. Trabajo práctico versus explicaciones & 3.61 & 3.65 & 3.65 & 3.96 & $\begin{array}{c}1,48 \\
(.220)\end{array}$ \\
\hline 2b. Trabajo práctico con instrucciontes & 3.95 & 3.80 & 3.84 & 3.56 & $\begin{array}{c}1.62 \\
(.184)\end{array}$ \\
\hline 3a. Oportunidad para seguìr su iniciabiva & 3.73 & 3.89 & 4.08 & 4.17 & $\begin{array}{c}3.44 \\
(017)\end{array}$ \\
\hline 4a. Trabajo individualizacto & 2.67 & 2.79 & 2.81 & 2.50 & $\begin{array}{c}1.33 \\
(.264)\end{array}$ \\
\hline 4b. Trabajo en pequeños gnupos & 3.95 & 3.88 & 3.84 & 4.21 & $\begin{array}{c}1.69 \\
(.169)\end{array}$ \\
\hline Sa. Evaluación constante por el profesor & 3.73 & 3.87 & 3.95 & 3.95 & $\begin{array}{c}1.44 \\
(.230)\end{array}$ \\
\hline Sb. Reachazo a ser evaluado & 3.45 & 3.29 & 3.39 & 3.39 & $\begin{array}{c}2.78 \\
(.097)\end{array}$ \\
\hline
\end{tabular}

- Número de alumnos. Las preferencias medias estan basadas en una escala de cinco juntos, que varia desde $5=$ alta preferencia hasta $]=$ =aja preferencia. 
éxito. Estos hechos, analizados conjuntamente, nos pueden conducir a previsiones contradictorias sobre la forma en que este tipo de alumnos responderán a diferentes situaciones en el aula.

Los resultados obtenidos en el presente estudio indican una preferencia de estos alumnos por un cierto riesgo, el cual suele encerrar las estrategias elegidas por ellos frente a aquellas otras totalmente centradas en el profesor. No obstante el grado de definición frente a los métodos de enseñanza es mucho menor al presentado por el resto de los modelos motivacionales, y está en disonancia con lo descrito en la Iiteratura. Hofstein y Kempa (1985) indican que estos alumnos deberían preferir estrategias expositorias, ya que éstas suelen ir asociadas con unos objetivos claros y precisos, y también una evalución constante por parte del profesor.

Esta disonancia pide una explicación; una posible, que a nuestros ojos es bastante plausible, es que alumnos que están comprometidos en un sistema competitivo'de educación y motivados por la obtención de éxito, pierden interés en las estrategias en sí mismas. Su importancia radica en ser únicamente medios para lograr el éxito buscado; y esto puede depender de las diferentes circunstancias, e incluso de los profesores que estén en juego en cada momento. Si esto es cierto, estos alumnos aceptarán cualquier estrategia que les permita alcanzar éxito.

\section{CONCLUSIONES}

La existencia de los cuatro modelos motivacionales descritos por Adar (1969), que muestran sus preferencias y rechazos por diferentes estrategias didácticas de la enseñanza de las Ciencias, es, según nuestra opinión, un importante punto de reflexión. En este momento, en que el estudio de los esquemas conceptuales o ideas intuitivas de los alumnos es objeto de una gran atención en el campo de la investigación didáctica, nosotros propugnamos que si desde el punto de vista cognoscitivo es necesario tener en cuenta las ideas con que los alumnos llegan a las aulas, desde un punto de vista afectivo es necesario considerar sus características motivacionales, cómo características internas que son y que se traducen en diferentes preferencias por distintas estrategias de la enseñanza de las Ciencias. Lo que es válido para las variables cognoscitivas, debe ser igualmente válido para las variables afectivas.

Para el estudio de las aplicaciones e implicaciones educacionales de este hecho, es preciso examinar cómo nuestros resultados pueden relacionarse con:

- el proceso del aprendizaje

- Ia organización de la enseñanza.
Respecto al primer punto, es posible presuponer que una mejora del aprendizaje tendrá lugar cuando los alumnos sean expuestos a unas estrategias preferidas por ellos, ya que estas preferencias están basadas en sus características motivacionales internas. Aunque esta hipótesis no ha sido todavía demostrada experimentalmente, ya que constituye el siguiente paso de nuestro estudio, sin embargo, encuentra apoyo en todos los estudios llevados a cabo sobre la relación entre las estrategias didácticas de las Ciencias y estilos de aprendizaje (Smith y Renzulli 1984, Brophy y Good 1974, Hunt 1971). Por lo tanto, aunque en el momento presente carezcamos de evidencia empírica de cómo los resultados obtenidos pueden mejorar el aprendizaje de los alumnos, no deben ser ignorados, bien al contrario, deben ser tenidos en cuenta en el diseño y planificación de las experiencias del aprendizaje.

Con relación a la organización de las actividades de enseñanza, pensamos que es fundamental que los profesores consideren que se enfrentan a alumnos con diferentes características motivacionales en el momento de elegir la metodología a seguir en su clase. No estamos diciendo que las preferencias de los alumnos deban ser seguidas completamente, especialmente siendo una realidad que los profesores tienen que tratar con clases con un elevado número de alumnos; sin embargo, consideramos que una importante mejoría deberá producirse si se presta atención a la existencia de diferentes alumnos con diferentes características motivacionales.

Somos realmente conscientes de las dificultades reales que existen cuando entran en consideración las características individuales de los alumnos, pero desearíamos pedir a los profesores que usen un amplio espectro de estrategias didácticas, en lugar de limitarse al uso de una o dos de ellas. Nos sentimos solidarios con las palabras de Joyce y Hodges (1966): «Un profesor que puede exhibir una amplia gama de estilos de enseñanza es potencialmente más capaz de realizar su trabajo que uno cuyo repertorio es relativamente limitado».

Para acabar, queremos dedicar algunas palabras a lo que podríamos llamar «orientación» de las estrategias didácticas. Una misma estrategia puede sufrir diferentes orientaciones, por ejemplo, el aprendizaje por descubrimiento; éste puede estar orientado hacia la realización de actividades abiertas, puede ofrecer un alto grado de guía y dirección oly puede dirigirse hacia la posibilidad de trabajar en grupo. Según cada una de estas orientaciones, puede ser apropiado para los alumnos curiosos, los concienzudos o los sociables respectivamente.

Ausubel (1968) plantea que se averigue lo que el alumno sabe y que se le enseñe en consecuencia. Nosotros nos atreveríamos a añadir que se averiguen sus características motivacionales y que se utilicen las estrategias didácticas consecuentemente. 


\section{REFERENCIAS BIBLIOGRÁFICAS}

ADAR, L., 1969. A theoretical framework for the study of motivation in Education. (The Hebrew University, School of Education: Jerusalem).

AUSUBEL, D.P., 1963. The psychology of meaningful verbal learning. (Grune and Stratton: New York).

AUSUBEL, D.P., 1968. Educational Psychology: A cognitive view. (Holt, Rinehart and Winston: New York).

BROPHY, J.E y GOOD, T.L., 1974. Teacher-student relationships: Causes and consequences. (Holt, Rinehart and Winston: New York).

BRUNER, I.S., 1961. The act of discovery, Harvard Educational Review, 31, pp. 21-32.

CLIFFORD, M.M., 1988. Failure tolerance and academic risktaking in ten to twelve year old student, British Journal of Educational Psychology, 58, pp. 15-27.

DODGE, B.J., 1978. Toward a conceptual framework for motivational design, NSPI Journal, 17(6), pp. 8-10.

EGGLESTON, J.F., GALTON, M.J. y JONES, M.E., 1976. Processes and products of science teaching. (Macmillan Education: London).

GOOD, T.L. y POWER, C.N., 1976. Designing successful classroom environments for different types of students, Journal of Curriculum Studies, 8(1), pp. 45-60.

HEATHCOTE, G., KEMPA, R, y ROBERTS, I, 1982, Curriculum styles and strategies. (DES. Further Education, Curriculum Review and Development Unit: London).

HERMAN, G., 1969. Learning by discovery. A critical review of studies, The Journal of Experimental Education, $38(1)$, pp. $58-72$.

HOFSTEIN, A. y KEMPA, R.F., 1985. Motivating strategies in science education: Attempt at an analysis, European Journal of Science Education, 7(3), pp. 221-229.
HUNT, D.E., 1971. Matching models in education. The coordination of teaching methods with students characteristics. (Ontario Institute for Studies in Education, Monograph series N. 10: Canada).

JOYCE, B.R. y HODGES, R.E., 1966. Instructional flexibility training, Journal of Teacher Education, 17, pp. 409-416.

KAUFMAN, B.A., 1971. Psychological implications of discovery learning in science, Science Education, 55(1), pp. 7383.

KEMPA, R.F. y MARTÍN DÍAZ, M.J., 1990. Motivational traits and preferences for different instructional modes in science. Part I: Students' motivational traits, International Journal of Science Education (en prensa).

KUHL J. y BLANKENSHIP, V., 1979. Behavioural change in a constant environment: Shift to more difficult tasks with constant probability of success, Journal of Personality and Social Psychology, 37, pp. 551-563.

MARTÍN DÍAZ, M.J., 1989. Students' motivational patterns and preferences for different instructional modes in science education. (Master of Arts, University of Keele: Inglaterra).

MCCLELLAND, D.C., ATKINSON J.W. CLARK, R.W. y LOWELL, E.L., 1953. The achievement motive. (AppletonCentury-Crofts: New York).

McCLELLAND, D.C., 1989. Estudio de la motivación humana. (Narcea: Madrid).

ORBACH, E., 1979. Simulation games and motivation for learning. A theoretical framework, Simulation and Games, I0(1), pp. 3-40.

SCHNEIDER, K. y POSSE, N., 1982. Risk taking in achievement oriented situations: Do people really maximize affect or competence information?, Motivation and Emotion, 6, pp. 259.271

SMITH, L.H. y RENZULLI, J.S.,1984. Leaning style preferences: A practical approach for classroom teacher, Theory into practice, $23(1)$, pp. 44-50. 


\section{ANEXO}

1. Cuando estoy estudiando una asignatura de ciencias, si no entiendo tuna palabra la busco en el diccionarto.

2. Me preocupa mucho si veo el enunciado de un problema de Matemáticas en la pizarra y no sé hacerlo.

3. Me gusta aprender sobre los nuevos descubrimientos científicos.

4. No me importa trabajar duro (estudiar mucho) en Ias asignaturas de ciencias si al finai consigo unas buenas notas.

5. Es más importante tener amigos que estar entre los mejores de la clase.

6. Me gusta trabajar en grupo para conocer las opiniones de mis compañeros sobre temas científicos.

7. Me gusta tener nuevos profesores.

8. Me gusta estar entre los mejores de la clase.

9. Creo que es dificil saber lo que significan Ios resultados de los experimentos.

10. En el laboratorio de Química, me gusta mezclar diferentes sustancias para ver lo que pasa.

11. Cuando en clase tenemos que trabajar en grupo, me siento orgulloso si mis compañeros me eligen para trabajar conmigo.

12. No me gusta que mis compañeros sepan que he tenido una mala nota en Física.

13. Cuando no termino de hacer todos los problemas que tenía que hacer en casa la preocupación no me deja dormir.

14. Uno de mis propositos en el instituto es conocer cosas nuevas.

15. Creo que es divertido competir con los compañeros de clase por las mejores notas.

16. Me importa mucho lo que mis compañeros piensen de mí.

17. Si tengo que elegir a mis compañeros para trabajar en grupo, elijo a mis amigos.

18. No me gusta hacer los problemas de Física deprisa. Prefiero tomarme el tiempo necesario para hacerlos lo mejor posible.

19. Me gusta que el profesor me felicite delante de toda la clase por una buena nota obtenida.

20. Estoy interesado en muchos temas científicos que no se enseñan en el instituto.

21. Me preocupa más que un compañero se enfade conmigo que mis problemas de Física estén mal hechos.

22. Rechazo una invitación para salir con unos amigos si tengo que estudiar para un examen de Química que tendré dentro de una semana.

23. No me importa trabajar duro en las asignaturas de ciencias si al final consigo aprender.
24. Una de las cosas más importantes en el instituto es tener buenos amigos.

25. Me gusta comprobar las explicaciones del profesor mirando en diferentes libros.

26. En las discusiones en clase, me gusta sobresalir.

27. Si un compuñero me pregunta algo en un examen, intento ayudarle.

28. Cuando saco malas notas, me siento avergonzado.

29. Cuando hago exámenes la cuestión más importante para mí es sacar la mejor nota de la clase.

30. Cuando escucho al profesor hablamos sobre temas científicos, se me ocurten un montón: de preguntas que hacerle.

31. Me gusta que el profesor valore los esfuerzos que hago para resolver bien los problemas de ciencias.

32. Cuando el profesor nos pone problemas en la clase, me gusta ser el primero en terminarlos.

33. Nomalmente me siento impaciente por empezar una nueva lección de ciencias.

34. No me importa que mis compañeros me copien los problemas o el trabajo que yo he hecho.

35. Pongo mucha atención en lo que el profesor me dice, ya que no quiero hacer nada mal.

36. Me gusta tener amigos que estén orgullosos de mis notas.

37. Todos los días el profesor me tiene que corregir los problemas y ejercicios que he hecho en casa.

38. Cuando tengo que resolver un problema de Matemáticas, no me importa Ia nota que pueda obtener, sino llegar hasta la solución final.

39. Presto todos los libros y apuntes que mis compañeros me piden.

40. Si tengo la posibilidad de elegir compañeros para trabajar en grupo, elijo a los mejores de la clase.

41. Trato por todos los medios de agradar al profesor con mi trabajo.

42. Me gusta leer libros de ciencias que van más allá de lo que nos piden en clase.

43. No me gusta que comparen mis notas con las de los demás.

44. Me gustan las discusiones en clase sobre temas científicos planteados por los alumnos, porque me permiten conocer cosas nuevas y diferentes a las que encuentro en los libros de texto.

45. No entiendo a los compañeros que saben hacer un problema y no se Jo quieren explicar a los demás.

46. Cuando trabajamos en grupo, me gusta que mi grapo sea el mejor.

47. No estoy satisfecho con lo que sé ahora y quiero seguir aprendiendo cosas nuevas. 
48. Prefiero antes ser evaluado por mis compañeros que por el profesor.

49. Cuando el profesor me devuelve los exámenes corregidos, estoy más interesado en la nota que en los errores que haya podido cometer.

50. En las clases de ciencias, suelo estar callado y haciendo mi trabajo o escuchando al profesor.

51. Creo que trabajar en grupo es interesante, porque me da la posibilidad de relacionarme con mis compañeros.

52. Creo que es divertido tratar de explicar las leyes científicas de diferente forma a la utilizada por el profesor.

53. Pienso que el profesor tiene razón al decir que no quiere que le interrumpamos sus explicaciones con preguntas.

54. En las discusiones en clase, me gusta que mi opinion prevalezca sobre las demás.
55. Cuando tengo una mala nota me enfado ut poco, peroenseguida me digo a mí mismo que la proxima vez será mejor.

56. Me gusta poner mucha atención en mi trabajo, para hacerlo to mejor posible.

57. Me gustaría trabajar en grupo en las clases de ciencias, pero normalmente no soy bien acogido entre mis compañeros.

58. Creo que es interesante hacer problemas y ejetcicios en casa, porque de esta forma estoy seguro de aprender más.

59. Para salir voluntario a hacer un problema tengo que estar seguro de que sé hacerlo.

60. Cuando trabajamos en grupo, no me importa que mis compañeros del gnupo no sean los mejores de la clase. 